\title{
Seeking Europa's Ocean
}

\author{
Robert T. Pappalardo \\ Jet Propulsion Laboratory \\ California Institute of Technology \\ email: robert.pappalardo@jpl.nasa.gov
}

\begin{abstract}
Galileo spacecraft data suggest that a global ocean exists beneath the frozen ice surface Jupiter's moon Europa. Since the early 1970s, planetary scientists have used theoretical and observational arguments to deliberate the existence of an ocean within Europa and other large icy satellites. Galileo magnetometry data indicates an induced magnetic field at Europa, implying a salt water ocean. A paucity of large craters argues for a surface on average only $\sim 40-90$ Myr old. Two multi-ring structures suggest that impacts punched through an ice shell $\sim 20 \mathrm{~km}$ thick. Europa's ocean and surface are inherently linked through tidal deformation of the floating ice shell, and tidal flexing and nonsynchronous rotation generate stresses that fracture and deform the surface to create ridges and bands. Dark spots, domes, and chaos terrain are probably related to tidally driven ice convection along with partial melting within the ice shell. Europa's geological activity and probable mantle contact permit the chemical ingredients necessary for life to be present within the satellite's ocean. Astonishing geology and high astrobiological potential make Europa a top priority for future spacecraft exploration, with a primary goal of assessing its habitability.
\end{abstract}

Keywords. Europa, habitability, ocean, convection, ice

Four hundred years ago, the discovery of the four Galilean moons of Jupiter forever changed our sense of place in the Universe (Figure 1). No longer could humans claim to be at its center, and the Copernican Revolution was underway. Today one of those moons - Europa - has the potential to again revolutionize science and our sense of place in the Universe, if we were to find life there.

Beneath the icy surface of Europa may be the solar system's most promising location for extant life beyond Earth. As one of the most geophysically and astrobiologically fascinating bodies in our solar system, Europa's exploration is key in advancing our understanding of habitable zones in our solar system, and other solar systems. If Europa contains a global ocean within, its volume is expected to be 2-3 times the volume all of Earth's oceans. Here I review some of the history, evidence, and implications of a subsurface ocean within Europa.

\section{Oceans Come and Go}

In the 1970s, it was realized that internal heat might be sufficient to melt oceans within large icy moons such as Europa, at least early in solar system history (Consolmagno and Lewis; 1976; Fanale et al., 1977). However, soon afterward, Reynolds and Cassen (1979) realized that the icy shell which caps an internal ocean would thicken as the satellite lost heat over geological time, eventually reaching a critical thickness at which it would transition from conductive to convective heat loss. When convection occurs, warm ice at the base of a floating icy shell would move buoyantly upward, and cold ice near the top of the icy shell sinks downward. They estimated that solid-state convection of ice should cause a satellite to lose heat very rapidly, freezing an internal ocean in about 100 
million years. Even if Europa and other large icy satellites once had oceans, Reynolds and Cassen (1979) reasoned that they should be frozen solid today.

Later calculations indicated that tidal heating might change the story, for Europa at least. Cassen et al. (1982) and Squyres et al. (1983) argued that if Europa's icy shell is sufficiently warm and dissipative, then the icy shell might be so thin that tidal heating would not initiate. Thus, it seemed that Europa might generate enough internal heating as it is squeezed by Jupiter's gravity that it might maintain an ocean beneath its icy surface layer. The most detailed analyses were performed by Ojakangas and Stevenson (1989), who showed that Europa might possess a conductive icy shell that averages $\sim 20$ $25 \mathrm{~km}$ thick above a liquid water ocean.

Voyager images of Europa were limited to $\sim 2 \mathrm{~km} /$ pixel at best (Figure 2). They revealed tantalizing hints of an icy surface consisting of bright plains and darker mottled terrain, criss-crossed by mysterious ridges and wider bright and dark bands (Lucchitta and Soderblom, 1982). The bands of the bright plains showed evidence for lateral motions of the ice, with translations of $\sim 20 \mathrm{~km}$, with new material (presumed to be solid-state ice, but plausibly liquid water) filling the gaps (Schenk and McKinnon, 1989; Pappalardo and Sullivan, 1994). The origins of ridges and mottled terrain remained mysterious, but prescient inference by Lucchitta and Soderblom (1982) suggested that vertical tectonism and intrusion dominates Europa's geology. The age of Europa's surface remained fully unknown: only a handful of certain craters were observed, suggesting a very young surface; however, if large impact craters flattened through relaxation (Squyres et al., 1983)

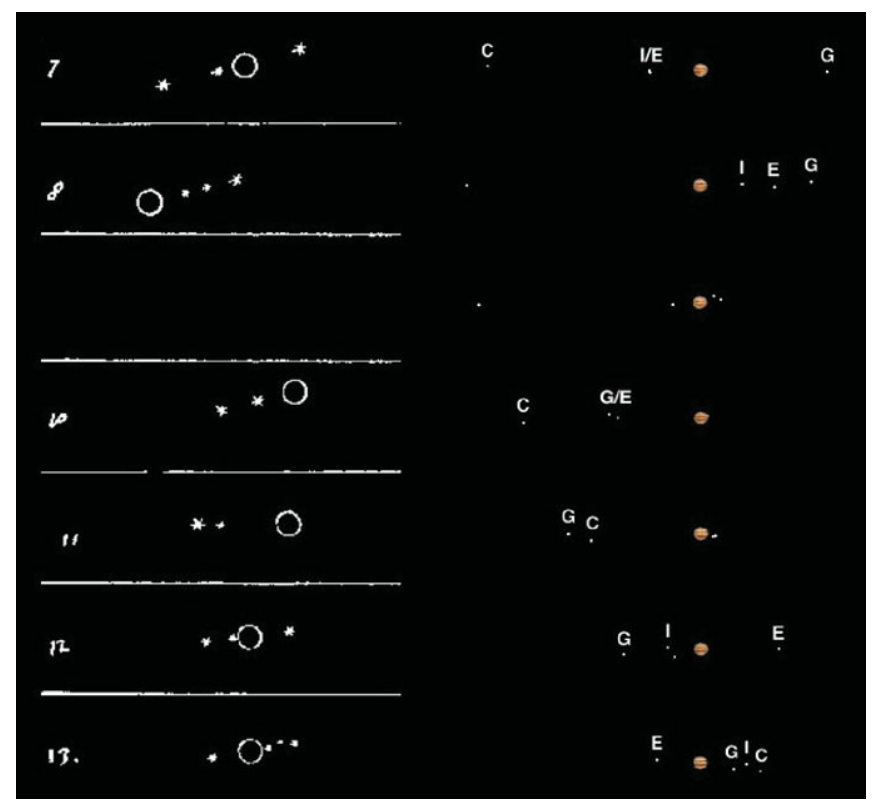

Figure 1. Galileo's sketches of the Medicean Stars, from January 7-13, 1610 (with the exception of Jan. 9, which was cloudy), compared to a simulated view of the Galilean satellites on the same nights from the Jet Propulsion Laboratory's Solar System Simulator (space.jpl.nasa.gov) viewed from Earth at about $1 \mathrm{hr}$ past the time of sunset in Padua, Italy. Labels indicate the positions of the satellites that Galileo identified: Io (I), Europa (E), Ganymede (G), and Callisto (C). On some nights, two satellites apparently were too close to each other or to Jupiter for Galileo to distinguish them as separate objects. Note that Europa and Io blended together into one point in the January 7 discovery sketch. 
and the small pits observed in the mottled terrain represented numerous small impact craters (Malin and Pieri, 1986) then the surface might be quite old.

\section{Europa's Ocean}

These uncertainties and predictions set the stage for the Galileo mission, which included 12 close fly-bys of Europa during the years 1996-2000 (Alexander et al., 2009). The results opened our eyes to the intricacies of Europa and the likelihood of an ocean within.

Doppler gravity data from the Galileo flybys indicates that Europa's interior is layered (Figure 3, inset), with an iron core and rocky mantle, topped by an $\mathrm{H}_{2} \mathrm{O}$-rich layer about $100 \mathrm{~km}$ thick (Schubert et al., 2009). However, gravity data alone cannot distinguish whether any of the $\mathrm{H}_{2} \mathrm{O}$ layer consists of liquid water.

It was the Galileo magnetometer measurements which provided most direct evidence for liquid water within Europa today, through indication of an induced magnetic field (Khurana et al., 2009). Jupiter's magnetic field is tilted by about $10^{\circ}$ compared to the equatorial plane in which Europa and the other Galilean satellites orbit. As Jupiter rotates with its rapid 10 hour period, its magnetic field rotates along with it. But because of the tilt, Jupiter's moons alternately find themselves above then below the magnetic equator of Jupiter. This means that the moons feel Jupiter's magnetic field alternating in polarity, cycling at the synodic period of the satellite relative to Jupiter as it rotates, i.e. $11.2 \mathrm{hr}$ for Europa.

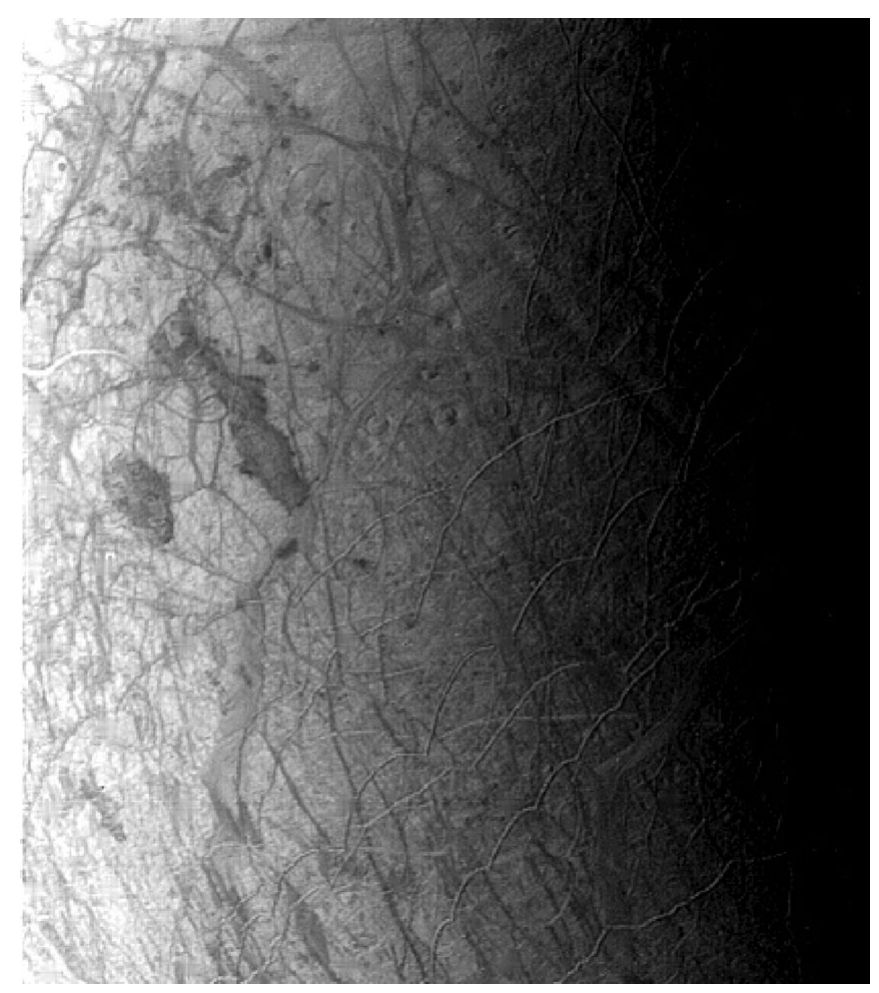

Figure 2. A portion of one of the best Voyager images of Europa at $\sim 2 \mathrm{~km} /$ pixel, showing a bewildering array of spots, ridges, bands, pits, and domes. Cycloidal ridges are prominent in the lower right portion of the scene. 
During Galileo's nominal mission, firm evidence arose for an induced field and thus a conducting layer at shallow depth within Callisto (Kivelson et al., 1999). The only reasonable conductive material is salt water, maintained by the shallow thermal gradient of the satellite, probably in combination with salt and/or ammonia antifreeze (Spohn and Schubert, 2003). An induced field was hinted at for Europa, but confirmation awaited the last close fly-by of Europa in 2000, when Galileo for the first time encountered Europa at close range while the moon was beneath Jupiter's magnetic equator (Kievelson et al., 2000). The data confirmed an induced magnetic field, indicating a subsurface ocean within the outer $30 \mathrm{~km}$ or so of the satellite (Zimmer et al., 2000). Ultimately an ocean was inferred within Ganymede as well, though that moon's intrinsic magnetic field makes it difficult to confidently tease out this result (Kivelson et al., 2002).

Unlike its larger sibling satellites Ganymede and Callisto, the relatively thin $\mathrm{H}_{2} \mathrm{O}$ layer at Europa means that pressures never get high enough for high-density ices to form beneath its ocean. This means that Europa's salty ocean is not sandwiched between ice layers as are the probable oceans of Ganymede and Callisto; instead Europa's ocean is expected to be in direct contact with a rocky mantle below (Schubert et al., 2009). A rocky seafloor means that the chemical nutrients that might support life can be supplied directly from the rocky mantle, making Europa astrobiologically attractive, as discussed below.

\section{Europa's Youthful Surface}

Europa's remarkable geology as revealed by Galileo spacecraft data indirectly tells of a subsurface ocean beneath (Figure 3). Most remarkable of all about the surface is its confirmed youth. There are only 23 known impact features larger than $10 \mathrm{~km}$ in diameter on the $\sim 75 \%$ of the surface sufficiently imaged to identify them (Schenk and Turtle, 2004). Combined with models of the flux of recent impactors at Europa, this implies that the surface is very young, with an average age of about 40-90 Myr, with an uncertainty of

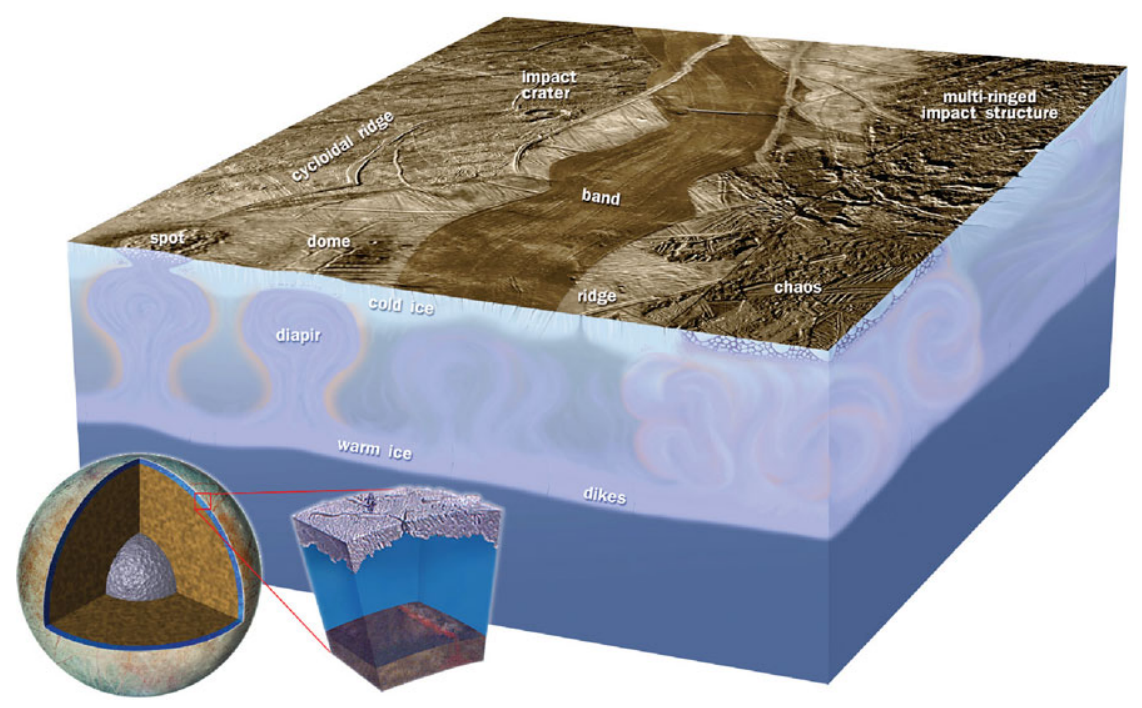

Figure 3. Schematic view of Europa's icy shell, illustrating selected geological features and their interpretation. Insets illustrate the likely deep interior structure of Europa, and the context of its icy shell and ocean floor. 
about a factor of 2 (Zahnle et al., 2003; Bierhaus et al., 2009). Much of Europa's surface activity has transpired in the $1 \%$ of Solar System history since dinosaurs populated the Earth. Such a geologically recent surface argues that if a subsurface ocean was involved in the formation of Europa's surface features, that ocean should still be present today.

Europa's impact structures hint of a warm icy lithosphere and an ocean below (Figure 4). Europa's largest impact craters, $\sim 26 \mathrm{~km}$ diameter Pwyll and $23 \mathrm{~km}$ diameter Manannán, are more shallow than similarly sized craters on the terrestrial planets or on most other icy satellites, suggesting that they impacted a warm icy lithosphere (Schenk and Turtle, 2009). Two features termed "multi-ring structures" are not morphologically craters but are certainly the sites of large impacts: they show numerous concentric rings, display disaggregated centers, and have radial chains of secondary craters (Moore et al., 1998, 2001). These multi-ring structures are believed to have penetrated through an ice shell about $20 \mathrm{~km}$ in thickness into liquid water below, resulting in their unusual morphologies (Schenk and Turtle, 2004).

Europa's troughs and ridges trace patterns that indirectly betray the existence of an ocean below. Most satellites are in synchronous rotation, having despun early in Solar System history such that they always show the same face to their parent planets as they orbit. However, a global ocean can permit the floating icy shell to slip relative to the satellite interior, undergoing "non-synchronous rotation," taking $\sim 10^{7}-10^{9} \mathrm{yr}$ for the icy shell to slip once around over the interior (Greenberg and Weidenshilling, 1984; Ojakangas and Stevenson, 1989). Whether significant stresses should result depends on the rotation rate relative to the relaxation rate (thus viscosity) of the icy shell (Wahr et al., 2009). Several researchers have compared the predicted pattern and distribution of non-synchronous rotation stresses to Europa's global-scale lineaments, concluding that this process has likely occurred through at least some portion of Europa's geologically recent history (Kattenhorn and Hurford, 2009; Selvans et al., 2010). There have also been

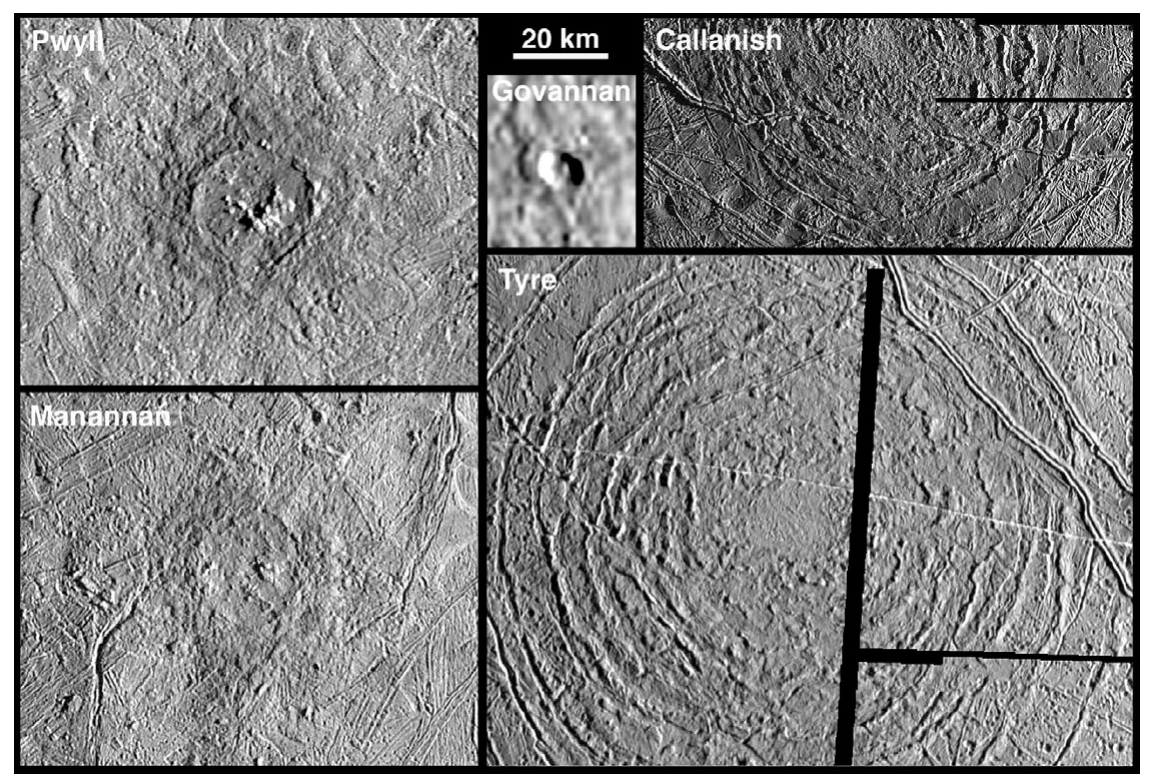

Figure 4. Europa's largest impact craters Pwyll and Manannán, and the multi-ring structures Callanish and Tyre, which probably formed by impacts that penetrated to Europa's ocean. The $11 \mathrm{~km}$ diameter crater Govannan has a more conventional morphology. 
suggestions that true polar wander of Europa's icy shell has occurred, flipping Europa's icy shell through time (e.g. Schenk et al., 2008).

Because Europa's orbit is somewhat elliptical, with an eccentricity of 0.0094 , it experiences a changing gravitational pull from its parent planet as it orbits Jupiter each Europa day (eurosol) of 3.55 days. This radial tide should cause Europa's icy shell to flex by about $30 \mathrm{~m}$ as it orbits, with slight variation in the predicted tidal amplitude depending on the thickness and rigidity of the ice shell (Moore and Schubert, 2000). A librational tide is also induced as Europa travels in its elliptical orbit. This tidal flexing means that the stresses at all non-equatorial points on the surface continually rotate direction and change magnitude as Europa orbits (Greenberg et al., 2002). Some of Europa's fractures and ridges trace out cycloidal patterns (Figure 2), displaying a series of arcs connected at sharp cusps; this unique pattern is probably related to propagation of cracks in the rotating and fluctuating stress environment (Hoppa et al., 1999). Indirectly, these cycloidal features betray a hidden ocean, because the stress required to form them would be too small if Europa has no ocean, as then the ice shell would flex by $<1 \mathrm{~m}$ (Hoppa et al., 1999; Moore and Schubert, 2000).

Europa's ubiquitous ridges typically have a distinctive double ridge morphology, with a valley in between (Figure 5). Formation of these ridges is probably related to the rise of water or warm ice along fractures (Kattenhorn and Hurford, 2009; Prockter and Patterson, 2009). A geophysically plausible model for their formation suggests that ridges are produced as the opposing sides of faults grind back-and-forth against each other as Europa orbits, generating heat akin to rubbing your hands together. This would warm the ice to either side sufficiently to induce buoyant uplift to form a ridge and/or would allow compressive stress to act on these weak plains, and might even generate enough heat to create melt along ridge axes (Nimmo and Gaidos, 2002). Subsequent shearing of these weak tabular zones might permit the formation of wider and more complexly lineated ridges (Aydin, 2006).

Many of Europa's wider dark and gray bands (Figure 6), which were first characterized in Voyager images, show fine striations along their lengths when seen at the higher resolutions that Galileo images provided (Sullivan et al., 1998). These may be places where the icy shell has spread apart along older ridges, exposing warm icy material

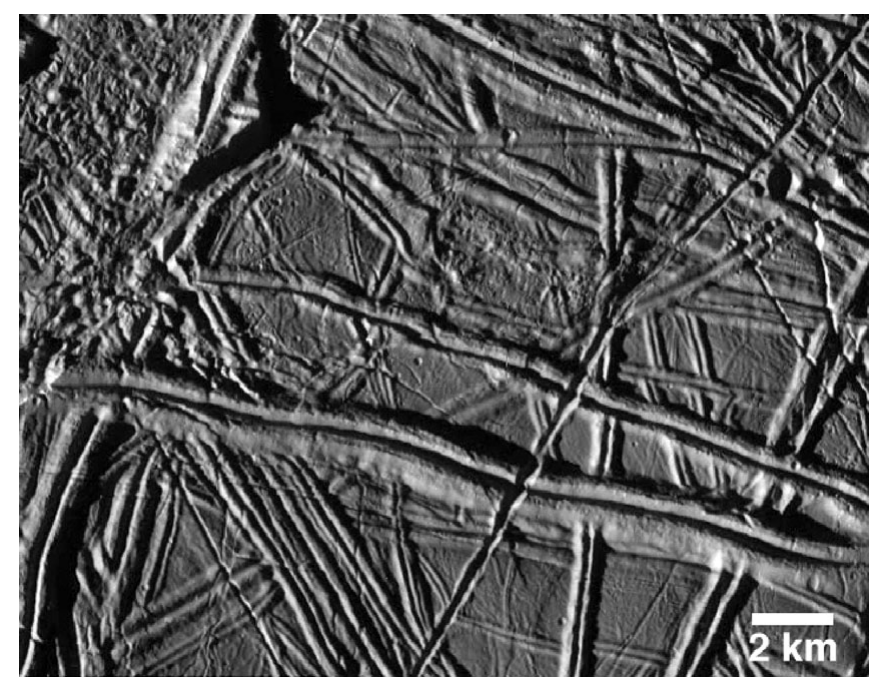

Figure 5. Typical double ridges on Europa's surface. 
from below, which is then fractured as extension continues (Prockter and Patterson, 2009). This model suggests that bands are icy analogs to the spreading centers that exist on Earth's rocky ocean floors, with new lithospheric ice created along an axial trough and shunted laterally then commonly faulted (Prockter et al., 2002). Unlike Earth's plate tectonics, however, there are no subduction zones identified on Europa, and only rare examples of folding have been identified (Prockter and Pappalardo, 2000), so the compensation of band extension remains an unresolved issue.

Wherever Europa's surface is somehow breached by geological activity to expose subsurface material, that material is relatively dark and a ruddy ochre in color, in contrast to the bright icy older surface. Multispectral characterization suggests that the colored materials contain hydrated sulfate salts and hydrated sulfuric acid (Dalton et al., 2007; Carlson et al., 2009). Salts may originate in the ocean (McCord et al., 1999; Zolotov and Kargel, 2009), while sulfuric acid hydrate may be a product of radiation-driven chemistry, radiolytically generated as high-energy charged particles of Jupiter's magnetosphere slam into Europa's surface. None of these materials is colored, but these same materials may gain their color from irradiation of sulfur-containing materials and growth of ochre-colored sulfur chains (Carlson et al., 2009).

Europa's face is freckled by pits, domes, and dark spots collectively known as "lenticulae," Latin for "freckles" (Carr et al., 1998; Pappalardo et al., 1998). Individual lenticulae are commonly about 5-10 km across, and some pepper the surface in clusters. They are probably the surface expression of upwelling warm ice diapirs, implying that Europa's icy shell is convecting (Barr and Showman, 2004), as Reynolds and Cassen (1979) had

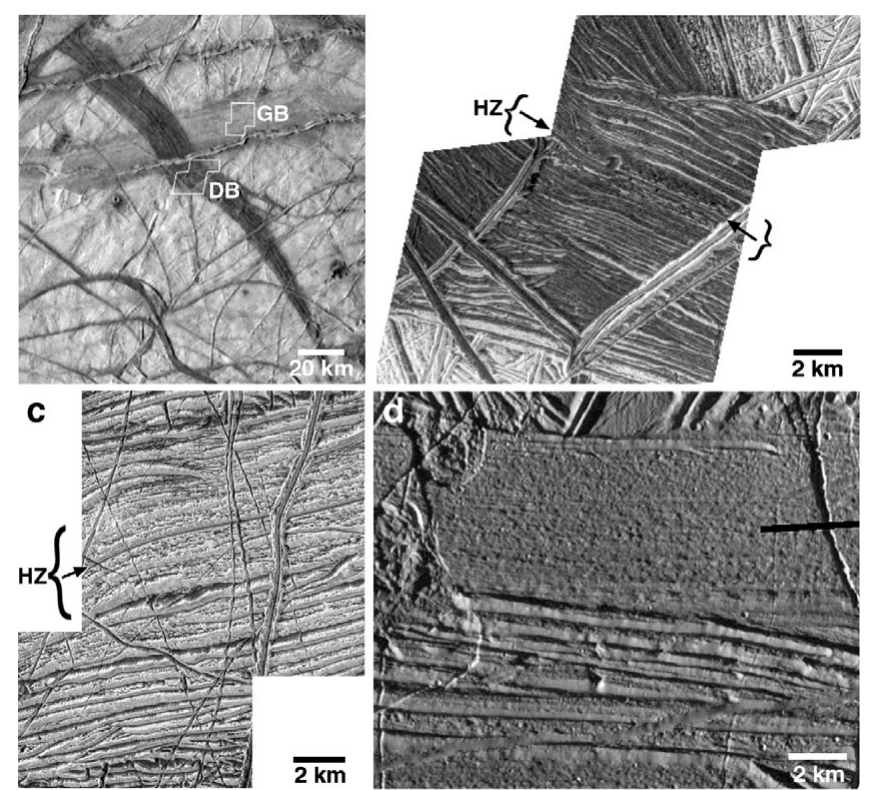

Figure 6. Examples of bands on Europa. (a) A dark wedge-shaped dark band (DB) cross-cuts a brighter (older) gray band (GB); outlines mark locations of the two subsequent views. (b) At high-resolution, the dark band shows multiple subparallel ridges and troughs that flank a central trough (arrows) and hummocky zone (braces, HZ). (c) At high resolution, the gray band shows similar characteristics of a central trough (arrow) and hummocky zone (braces, HZ). (d) A band seen at high resolution and high solar incidence angle displays subparallel ridge and troughs with characteristics of normal fault blocks. These characteristics have been compared to spreading centers on Earth. 
first predicted. This implies that diapirs of warm ice apparently risen up from the base of the floating icy shell, and some have impinged on the cold surface layer to warp it upward into domes, while others have pierced the cold surface to flow out onto the surface, though piercing the coldest near-surface ice implies additional processes such as compositional buoyancy of the diapirs (Pappalardo and Barr, 2004). Pits may be locations where warm diapirs have triggered melting within the ice shell, resulting in downwarping of the surface as melt drains downward in the ice shell below (Singer et al., 2010).

Europa shows large disrupted regions aptly termed "chaos," where the surface is broken into raft-like plates that have rotated and tilted in a sea of rubbly debris (Carr et al., 1998). Some argue these are places where the icy shell has completely melted, perhaps above a warm plume of water below (Greenberg et al., 1999); others infer that these are places where ice convection has been especially intense, disrupting the cold icy surface above, and partially melting near-surface ice to allow fragmented blocks to slide and turn (Collins et al., 2000). Either way, chaos tells of a warm and active icy shell, with at least partial melting of the ice (Collins and Nimmo, 2009).

Reynolds and Cassen (1979) predicted that convection should rapidly freeze an ocean, but the Galileo magnetometer data and the youthfulness of Europa's surface imply that Europa's ocean is still there. In recent decades, significant advances have been made in understanding of convection, tidal heating, and the properties of ice. Thus, it now seems that ice convection might not freeze an underlying ocean after all, but instead ice convection and an ocean can co-exist through time (Spohn and Schubert, 2003; Barr and Showman, 2004).

The thickness of Europa's icy shell is uncertain, but geological and geophysical modeling provides clues (Stevenson, 2000; Nimmo and Manga, 2009; McKinnon et al., 2009). Consideration of the amount of tidal and radiogenic heat from Europa predict an icy shell about 20 to $30 \mathrm{~km}$ thick (Hussman et al., 2002; Spohn and Schubert, 2003). Work using the most pertinent ice rheology and grain size data suggests that convection should initiate when Europa's ice shell is 15-25 km thick (McKinnon, 1999), so Europa's lenticulae are consistent with an icy shell $>15 \mathrm{~km}$ thick. Most telling, Europa's two largest bulls-eye-like multi-ringed structures indicate an icy shell about $20 \mathrm{~km}$ thick when they

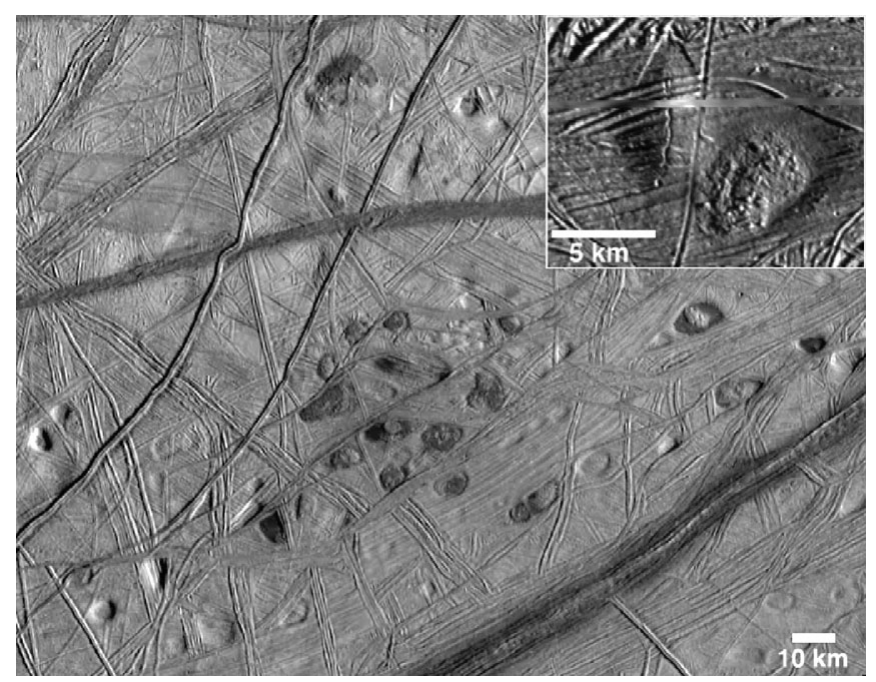

Figure 7. Pits, spots, and domes on Europa, commonly termed "lenticulae" in a regional view, and a high resolution view (inset). Illumination is from the east (right) in both images. 
formed in geologically recent times (Schenk, 2002). Some researchers have argued that Europa's ice shell might be only a few kilometers thick (Greenberg et al., 2002); however, such a model is inconsistent with thermal, convection, and impact modeling. Instead, an icy shell $\sim 20 \mathrm{~km}$ thick is consistent with all of the geological evidence, while providing sufficient tidal amplitude to permit the formation of Europa's ubiquitous tectonic features.

\section{Europa and the Search for Life}

On Earth, everywhere there is water, there is life, so it is reasonable that the search for life in our solar system focuses on the search for water. Europa's probable ocean suggests is an important destination in the search for life in our Solar System. But life needs more than just water to exist, it also needs the chemical elements from which organic molecules can be built and a source of energy to power metabolism.

In the lightless depths of Europa, photosynthesis is an unlikely metabolic mechanism. Instead, it is chemosynthetic life which might plausibly exist, if the chemical disequilibrium to power metabolic reactions exists in Europa's ocean (Hand et al., 2009). In fact, Europa's intense geological activity and relatively thin icy shell combine to give its ocean advantages for habitability that other icy satellite oceans in our Solar System do not have. Geological breaching of Europa's icy shell combined with partial melting and convective transport could permit the ocean and surface to exchange water and ice, providing important advantages to life and the search for life.

The chemical energy for life could enter Europa's ocean from above and from below. Europa's surface is bathed in intense radiation from high-energy charged particles trapped in Jupiter's magnetosphere. These particles slam into Europa's ice-rich surface, transforming some of its $\mathrm{H}_{2} \mathrm{O}$ into molecules of oxygen $\left(\mathrm{O}_{2}\right)$, peroxide $\left(\mathrm{H}_{2} \mathrm{O}_{2}\right)$, and other oxidized compounds (Johnson et al., 2004; Paranicas et al., 2004). If these oxidants can be transported to the subsurface ocean by geological processes, they would make an ideal chemical fuel for life.

Meanwhile, if Europa's rocky mantle is hot, then reduced chemical nutrients could pour into the base of the ocean from below, potentially at black smokers analogous to those

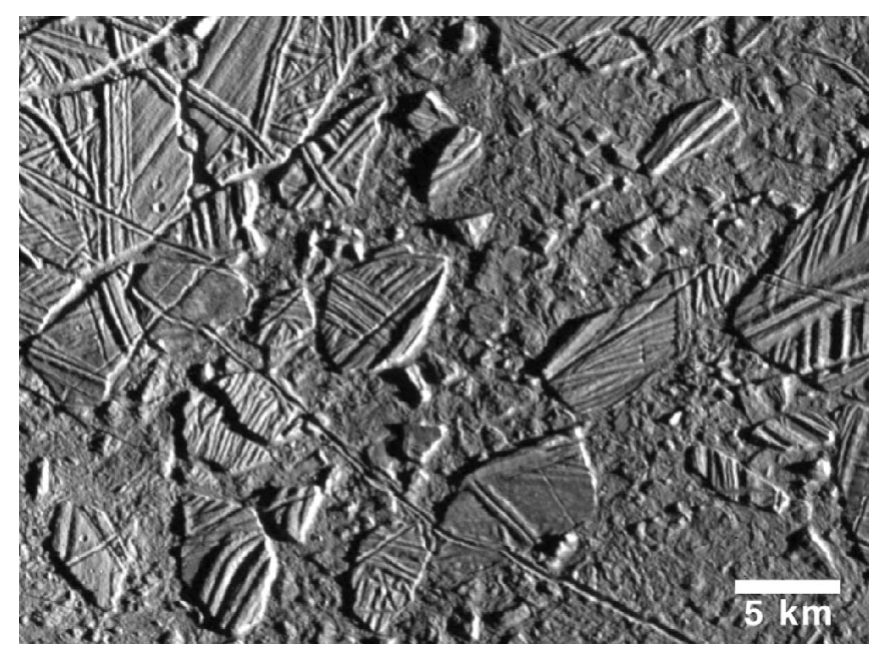

Figure 8. Chaos terrain on Europa, where city-sized icy blocks have rotated and translated in a matrix of disrupted and crumbled blocks. 
comprising hydrothermal vents that power communities of organisms on the terrestrial ocean floor (McCollom, 1999). Whether Europa's mantle is significantly tidally heated is currently unknown, and depends on the complex thermal-orbital evolution of Europa and the other Galilean satellites (Moore and Hussman, 2009).

If Europa's icy shell is only several kilometers thick and can completely melt through in places, then oxidants could be directly delivered to the ocean (Greenberg et al., 2000). However, in the more likely scenario where Europa's icy shell is $\sim 20 \mathrm{~km}$ thick, then convective activity and partial melting could transport surface oxidants to the ocean. Partial melting in chaotic terrain, in lenticulae, and along ridge axes may allow melt to sink downward through the cold near-surface ice and to the convective zone below on a time scale of $\sim 10-100 \mathrm{yr}$ (Barr et al., 2002). In turn, convection can circulate material from the top to bottom of the convective zone on a time scale of $\sim 10^{5}$ yr (Pappalardo and Barr, 2004). Through this indirect route, oxidants may be supplied to Europa's ocean below. In addition, some oxidants can be generated within the ice shell and ocean

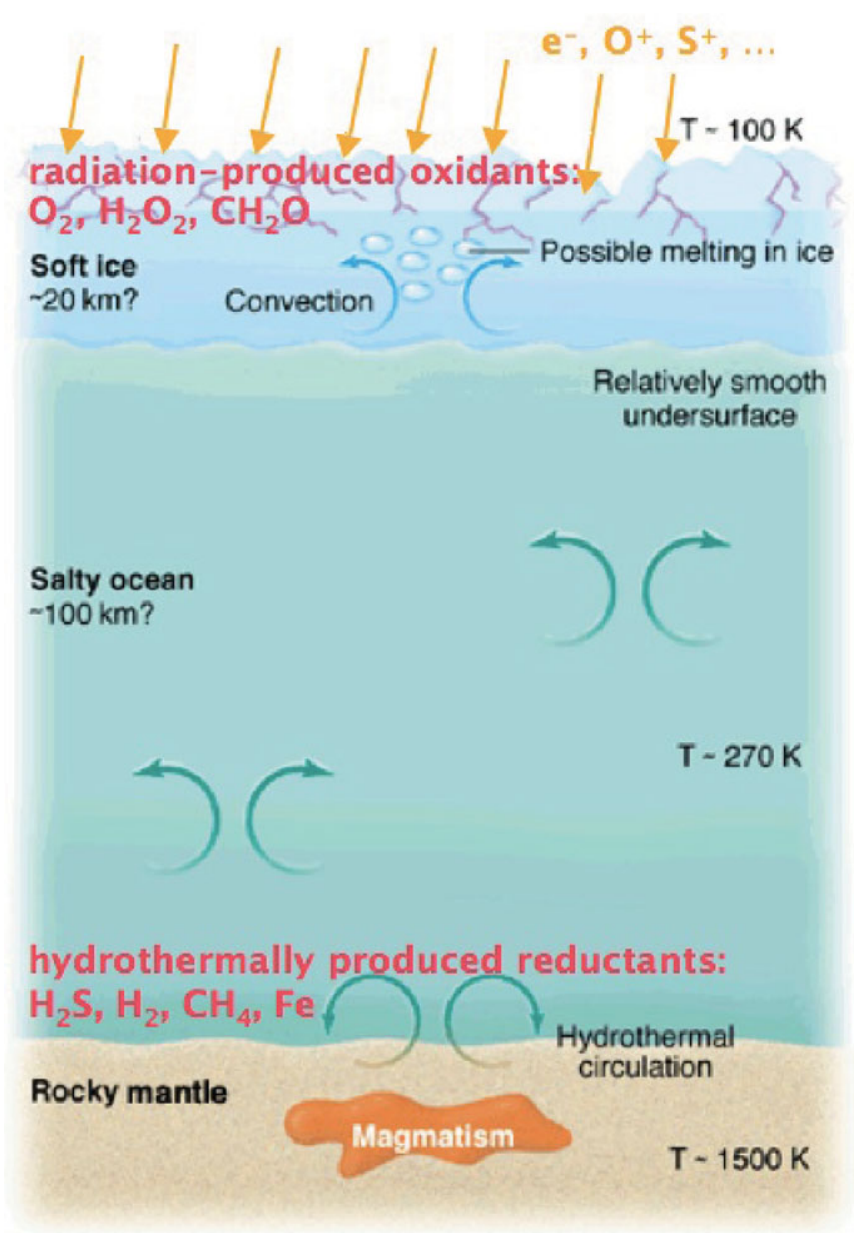

Figure 9. Representation of Europa's ice shell, ocean, and rocky mantle (after Stevenson, 2000). Oxidants are produced at Europa's surface by ion irradiation (arrows). Reductants might be produced at the ocean floor if the mantle rock is hot, promoting hydrothermal circulation. Such oxidants and reductants could serve to fuel life in Europa's ocean. 
by radioactive decay (Hand et al., 2007). Understanding transport mechanisms within Europa's icy shell is key to understanding the habitability of Europa's ocean.

The search for life at Europa will begin slowly, and requires patience. The Jupiter Europa Orbiter spacecraft presently on the drawing boards as part of the international Europa Jupiter System Mission is the next required step in evaluating the potential habitability of Europa (Greeley et al., 2009). Such an orbiting spacecraft would be able to characterize Europa's ocean, including its thickness and salinity, using the techniques of gravity and altimetry to measure Europa's tidal deformation, and magnetometry to much better characterize the satellite's induced magnetic field signature. Ice-penetrating radar could search for pockets of shallow water within the icy shell such as in chaos regions and along ridges, and could be correlated to geological indicators to understand surface-ice-ocean material exchange processes; a radar signal through sufficiently cold ice might even be able to directly detect the ocean (Blankenship et al., 2009). Compositional measurements, imaging, and charged particle readings could combine to indicate how Europa's surface features are created and evolve, and whether the surface chemistry is indicative of ocean chemistry, and whether that chemistry is conducive to life. The Jupiter Europa Orbiter could launch as soon as 2020, reach Jupiter by 2025, and enter Europa orbit in 2028.

In the more distant future, humans may land a spacecraft on Europa. Life cannot survive on Europa's surface because of the intense radiation by Jupiter's magnetospheric charged particles, but signs of life might be sought just tens of centimeters below the surface, where ice would protect any organic materials that might be dredged from the ice-ocean interface to near the surface. Signs of life might be detectable with a landed spacecraft which would use sophisticated chemical analyses and a simple microscope to search (Hand et al., 2009).

Europa is an astrobiologically and geophysically fascinating world, arguably offering the best prospect for present-day life in our Solar System beyond Earth. Europa's warm, salty ocean is in direct contact with a mantle that could be geologically active, supplying chemical nutrients from below, while its icy shell is thin and its surface is youthful, so recent or current activity could churn nutrients downward into the ocean. If life exists at Europa, its signs could be exposed at or near the surface.

More than four hundred years after its discovery, Europa may some day again bring about a revolution in scientific thought, and - if life is found there - may again change our sense of place in the Universe.

Acknowledgements This work was carried out at the Jet Propulsion Laboratory, California Institute of Technology, under a contract with the National Aeronautics and Space Administration.

\section{References}

Alexander, C., Carlson, R., Consolmagno, G., Greeley, R., \& Morrison, D. 2009, The exploration history of Europa Europa, R. T. Pappalardo et al., eds., pp. 3-26. Univ. of Arizona Press, Tucson

Aydin, A. 2006, Failure modes of the lineaments on Jupiter's moon, Europa: Implications for the evolution of its icy crust, J. Struct. Geol., 28, 2222-2236

Barr, A. C., Nimmo, F., Pappalardo, R. T., \& Gaidos, E. 2002, Shear heating and solid-state convection: Implications for astrobiology, Lunar Planet. Sci. Conf. XXXIII, abstract \#1545

Barr, A. C. \& Showman, A. P. 2009, Heat transfer in Europa's icy shell, Europa, R. T. Pappalardo et al., eds., pp. 405-430. Univ. of Arizona Press, Tucson 
Blankenship, D. D., Young, D. A., Moore, W. B., \& Moore, J. C. 2009, Radar sounding of Europa's subsurface properties and processes: The view from Earth, Europa, R. T. Pappalardo et al., eds., pp. 631-654. Univ. of Arizona Press, Tucson

Bierhaus, E. B., Zahnle, K., \& Chapman, C. R. 2009, Europa's crater distributions and surface ages, Europa, R. T. Pappalardo et al., eds., pp. 161-180. Univ. of Arizona Press, Tucson

Carlson, R. W., Calvin, W. M., Dalton, J. B., Hansen, G. B., Hudson, R. L., Johnson, R. E., McCord, T. B., \& Moore, M. H. 2009, Europa's surface composition, Europa, R. T. Pappalardo et al., eds., pp. 283-328. Univ. of Arizona Press, Tucson

Carr, M. H., Belton, M. J. S., Chapman, C. R., Davies, M. E., Geissler, P., Greenberg, R., McEwen, A. S., Greeley, R., Sullivan, R., Head, J. W., Pappalardo, R. T., Klaasen, K. P., Johnson, T. V., Moore, J., Neukum, G., Schubert, G., Burns, J. A., Thomas, P., \& Veverka, J. 1998, Evidence for a subsurface ocean on Europa, Nature, 391, 363-365

Cassen, P. M., Peale, S. J., \& Reynolds, R. T. 1982, Structure and thermal evolution of the Galilean satellites, Satellites of Jupiter, D. Morrison, ed., pp. 93-1128, Univ. of Ariz. Press, Tucson

Collins, G. \& Nimmo, F. 2009, Chaos on Europa, Europa, R. T. Pappalardo et al., eds., pp. 259-282. Univ. of Arizona Press, Tucson

Collins G. C., Head, J. W., Pappalardo, R. T., \& Spaun, N. A. 2000, Evaluation of models for the formation of chaotic terrain on Europa, J. Geophys. Res., 105, 1709-1716

Consolmagno, G. J. \& Lewis, J. S. 1976, Structural and thermal models of icy Galilean satellites, Jupiter, (T. A. Gehrels, ed.), pp. 1035-1051. Univ. of Arizona, Tucson

Dalton, J. B. 2007, Linear mixture modeling of Europa's nonice material using cryogenic laboratory spectroscopy, Geophys. Res. Lett.34, L21205, doi: 10.1029/2007GL031497

Fanale F. P., Johnson, T. V., \& Matson, D. L., 1977, Io's surface and the histories of the Galilean satellites, Planetary Satellites, (J. A. Burns, ed.), pp. 379-405. Univ. of Arizona, Tucson

Greeley, R., Pappalardo, R. T., Prockter, L. M., \& Hendrix, A. 2009, Future exploration of Europa, Europa, (J. A. Burns, ed.), (R. T. Pappalardo et al., eds.), pp. 655-695, Univ. of Arizona, Tucson

Greenberg, R. \& Weidenschilling, S. J. 1984, How fast do Galilean satellites spin?, Icarus, 58, $186-196$

Greenberg R., Hoppa, G. V., Tufts, B. R., Geissler, P., Riley, J., \& Kadel, S. 1999, Chaos on Europa, Icarus, 141, 263-286

Greenberg, R., Geissler, P., Tufts, B. R., \& Hoppa, G. V. 2000, Habitability of Europa's crust: The role of tidal-tectonic processes, J. Geophys. Res., 105, 17, 551-17, 562

Greenberg, R., Geissler, P., Hoppa, G., \& Tufts, B. R. 2002, Tidal tectonic processes and their implications for the character of Europa's icy crust, Rev. Geophysics 40, 1004, doi:10.1029/2000RG000096

Hand, K. P., Carlson, R. W., \& Chyba, C. F. 2007, Energy, chemical disequilibrium, and geological constraints on Europa, Astrobiology, 7, 1-18

Hand, K. P., Chyba, C. F., Priscu, J. C., Carlson, R. W., \& Nealson, K. H. 2009, Astrobiology and the potential for life on Europa, Europa, R. T. Pappalardo et al., eds., pp. 589-630. Univ. of Arizona Press, Tucson

Hoppa, G. V., Tufts, B. R., Greenberg, R., \& Geissler, P. E. 1999, Formation of cycloidal features on Europa, Science, 285, 1899-1902

Hussman, H., Spohn, T., \& Wieczerkowski, K. 2002, Thermal equilibrium states of Europa's ice shell: Implications for internal ocean thickness and surface heat flow, Icarus, 156, 143-151

Johnson, R. E., Burger, M. H., Cassidy, T. A., Leblanc, F., Marconi, M., \& Smyth, W. H. 2009, Composition and detection of Europa's sputter-induced atmosphere, Europa, R. T. Pappalardo et al., eds., pp. 507-528. Univ. of Arizona Press, Tucson

Kattenhorn, S. A. \& Hurford, T. 2009, Tectonics of Europa, Europa, R. T. Pappalardo et al., eds., pp. 199-236. Univ. of Arizona Press, Tucson

Khurana, K. K., Kivelson, M. G., Hand, K. P., \& Russell, C. T. 2009, Electromagnetic induction from Europa's ocean and the deep interior, Europa, R. T. Pappalardo et al., eds., pp. 571587. Univ. of Arizona Press, Tucson

Kivelson, M. G., Khurana, K. K., Stevenson, D. J., Bennett, L., Joy, S., Russell, C. T., Walker, R. J., Zimmer, C., \& Polansky, C. 1999, Europa and Callisto: Induced or intrinsic fields in a periodically varying plasma environment, J. Geophys. Res., 104 4609-4625 
Kivelson, M. G., Khurana, K. K., Russell, C. T., Volwerk, M., Walker, R. J., \& Zimmer, C. 2000, Galileo magnetometer measurements strengthen the case for a subsurface ocean at Europa, Science, 289, 1340

Kivelson, M. G., Khurana, K. K., \& Volwerk, M. 2002, The permanent and inductive magnetic moments of Ganymede, Icarus, 157, 507-522

Lucchitta, B. K. \& Soderblom, L. A. 1982, The geology of Europa, The Satellites of Jupiter, (D. Morrison, ed.), pp. 521-555. Univ. of Arizona, Tucson, Arizona

Malin, M. C. \& Pieri, D. C. 1986, Europa, Satellites, (J. A. Burns and M. S. Matthews, Eds.), pp. 689-716. Univ. of Arizona Press, Tucson

McCollom, T. M. 1999, Methanogenesis as a potential source of chemical energy for primary biomass production by autotrophic organisms in hydrothermal systems on Europa, J. Geophys. Res, 104, 30729-30742

McCord, T. B., Hansen, G. B., Matson, D. L., Johnson, T. V., Crowley, J. K., Fanale, F. P., Carlson, R. W., Smythe, W. D., Martin, P. D., Hibbitts, C. A., Granahan, J. C., Ocampo, A., \& the NMS Team 1999, Hydrated salt minerals on Europa's surface from the Galileo NIMS investigation, J. Geophys. Res, 104, 11827-11851

McKinnon, W. B. 1999, Convective instability in Europa's floating ice shell, Geophys. Res. Lett, 26, 951-954

McKinnon, W. B., Pappalardo, R. T., \& Khurana, K. K. 2009, Europa: Perspectives on an ocean world, Europa, (R. T. Pappalardo et al., eds.), pp. 697-709, Univ. of Arizona, Tucson

Moore, W. B. \& Schubert, G. 2000, The tidal response of Europa, Icarus, 147, 317-319

Moore, W. B. \& Hussman, H. 2009, Thermal evolution of Europa's silicate interior, Europa, R. T. Pappalardo et al., eds., pp. 369-380. Univ. of Arizona Press, Tucson

Moore J. M., Asphaug, E., Sullivan, R. J., Klemaszewski, J. E., Bender, K. C., Greeley, R., Geissler, P. E., McEwen, A. S., Turtle, E. P., Phillips, C. B., Tufts, B. R., Head, J. W., Pappalardo, R. T., Jones, K. B., Chapman, C. R., Belton, M. J. S., Kirk, R. L., \& Morrison, D. 1998, Large impact features on Europa: Results of the Galileo nominal mission, Icarus, $135,127-145$

Moore J. M., Asphaug, E. B., Michael, J. S., Bierhaus, B., Breneman, H. H., Brooks, S. M., Chapman, C. R., Chuang, F. C., Collins, G. C., Giese, B., Greeley, R., Head, J. W., Kadel, S., Klaasen, K. P., Klemaszewski, J. E., Magee, K. P., Moreau, J., Morrison, D., Neukum, G., Pappalardo, R. T., Phillips, C. B., Schenk, P. M., Senske, D. A., Sullivan, R. J., Turtle, E. P., \& Williams, K. K. 2001, Impact features on Europa: Results of the Galileo Europa Mission (GEM)., Icarus, 151, 93-111

Nimmo, F. \& Gaidos, E. 2002, Strike-slip motion and double ridge formation on Europa, J. Geophys. Res., 107, 1-8

Nimmo, F. \& Manga, M. 2009, Geodynamics of Europa's icy shell, Europa, (R. T. Pappalardo et al., eds.), pp. 381-404, Univ. of Arizona, Tucson

Ojakangas, G. W. \& Stevenson, D. J. 1989, Thermal state of an ice shell on Europa, Icarus, 81, $220-241$

Pappalardo, R. T. \& Barr, A. C. 2004, Origin of domes on Europa: The role of thermally induced compositional buoyancy, Geophys. Res. Lett., 31, L01701, doi:10.1029/2003GL019202

Pappalardo, R. T. \& Sullivan, R. J. 1996, Evidence for separation across a gray band on Europa, Icarus, 123, 557-567

Pappalardo, R. T., Head, J. W., Greeley, R., Sullivan, R. J., Pilcher, C., Schubert, G., Moore, W., Carr, M. H, Moore, J. M., Belton, M. J. S., \& Goldsby, D. L. 1998, Geological evidence for solid-state convection in Europa's ice shell, Nature, 391, 365-368

Paranicas, C., Cooper, J. F., Garrett, H. B., Johnson, R. E., \& Sturner, S. J. 2009, Europa's radiation environment and its effects on the surface, Europa, R. T. Pappalardo et al., eds., pp. 529-544. Univ. of Arizona Press, Tucson

Prockter, L. M. \& Pappalardo, R. T. 2000, Folds on Europa: Implications for crustal cycling and accommodation of extension, Science, 289, 941-943

Prockter, L. M. \& Patterson, G. W. 2009, Morphology and evolution of Europa's ridges and bands, Europa, (R. T. Pappalardo et al., eds.), pp. 237-258, Univ. of Arizona, Tucson

Prockter L. M., Head, J., Pappalardo, R., Sullivan R., Clifton, A. E., Giese, B., Wagner, R., \& Neukum, G. 2002, Morphology of europan bands at high resolution: A mid-ocean ridgetype rift mechanism, J. Geophys. Res., 107, 1-26 
Reynolds, R. T. \& Cassen, P. 1979, On the internal structure of the major satellites of the outer planets, Geophys. Res. Lett., 6, 121-124

Schenk, P. M. 2002, Thickness constraints on the icy shells of the Galilean satellites from a comparison of crater shapes, Nature, 417, 419-421

Schenk, P. \& McKinnon, W. B. 1989, Fault offsets and lateral crustal movement on Europa: Evidence for a mobile ice shell, Icarus, 79, 75-100

Schenk, P. M. \& Turtle, E. P. 2009, Europa's impact craters: Probes of the icy shell, Europa, R. T. Pappalardo et al., eds., pp. 181-198. Univ. of Arizona Press, Tucson

Schenk P., Matsuyama, I., \& Nimmo, F. 2008, True polar wander on Europa from global-scale small-circle depressions, Nature, 453, 368-371

Schubert, G., Sohl, F., \& Hussman, H. 2009, Thermal evolution of Europa's silicate interior, Europa, R. T. Pappalardo et al., eds., pp. 353-368. Univ. of Arizona Press, Tucson

Selvans, Z. A., Wolf, A. S., \& Pappalardo, R. T. 2010, A critical comparison of Europa's lineaments to non-synchronous rotation stresses, J. Geophys. Res.

Singer, K. N., McKinnon, W. B., \& Schenk, P. M. 2010, Pits, spots, uplifts, and small chaos regions on Europa: Evidence for diapiric upwelling from morphology and morphometry, Lunar Planet. Sci. Conf. 41, Abstract \#2195

Spohn, T. \& Schubert, G. 2003, Oceans in the icy Galilean satellites of Jupiter?, Icarus, 161, $456-467$

Squyres S. W., Reynolds, R. T., Cassen, P., \& Peale, S. J. 1983, Liquid water and active resurfacing of Europa, Nature, 301, 225-226

Stevenson, D. 2000, Europa's Ocean: The case strengthens, Science, 289, 1305-1307

Sullivan, R. \& 12 colleagues 1998, Episodic plate separation and fracture infill on the surface of Europa, Nature, 391, 371-373

Wahr, J., Selvans, Z. A., Mullen, M. E., Barr, A. C., Collins, G. C., Selvans, M. M., \& Pappalardo, R. T. 2009, Modeling stresses on satellites due to nonsynchronous rotation and orbital eccentricity using gravitational potential theory, Icarus, 200, 188-206

Zimmer C., Khurana, K. K., \& Kivelson, M. G. 2000, Subsurface oceans on Europa and Callisto: Constraints from Galileo magnetometer observations, Icarus, 147, 329-347

Zahnle, K., Schenk, P., Levison, H. F., \& Dones, L. 2003, Cratering rates in the outer solar system, Icarus, 163, 263-289

Zolotov, M. Yu \& Kargel, J. S. 2009, On the chemical composition of Europa's icy shell, ocean, and underlying rocks, Europa, R. T. Pappalardo et al., eds., pp. 431-458. Univ. of Arizona Press, Tucson 